\title{
ON SUBSETS WITH ASSOCIATED COMPACTA IN DISCRETE ABELIAN GROUPS
}

\author{
RON C. BLEI
}

ABstract. Let $\Gamma$ be a discrete abelian group. We prove that every non-Sidon set in $\Gamma$ contains $F$, a non-Sidon set with the property that for every $\varepsilon>0$ and compact set $K \subset \hat{\Gamma}$ with nonempty interior, there exists a finite set $\Lambda(\varepsilon, K) \subset F$, so that

$$
\sup _{x \in K}|p(x)| \geqq(1-\varepsilon)\|p\|_{\infty}, \quad \text { for all } p \in C_{E \backslash \Lambda}(\hat{\Gamma}) \text {. }
$$

In this paper we demonstrate that the necessary condition for Sidon sets in discrete, torsion free abelian groups, obtained by $M$. DéchampsGondim in [2], (Theorem A), fails in a strong way to be sufficient (Theorem B).

In what follows below, $\Gamma$ is a discrete abelian group, and $\hat{\Gamma}=G$. We refer to Chapters 1 and 2 of [6] for standard notation and facts. $E \subset \Gamma$ is a Sidon set if there exists $\alpha>0$ so that $\|p\|_{\infty} \geqq \alpha \sum_{\gamma \in \Gamma}|\hat{p}(\gamma)|$, for all trigonometric polynomials $p \in C_{E}(G)=\{f \in C(G): \hat{f}(\gamma)=0$ for $\gamma \notin E\}$.

Definition 1. $E \subset \Gamma$ is said to satisfy property (D) if for every $K \subset G$, a compact set with nonempty interior, there exists a finite set $\Lambda \subset E$, and $C>0$, so that

$$
\sup _{x \in K}|p(x)| \geqq C\|p\|_{\infty}, \quad \text { for all } p \in C_{E \backslash \Lambda}(G) .
$$

DEFINITION 2. $E \subset \Gamma$ is said to satisfy property (D1) if for every $\varepsilon>0$ and compact set $K \subset G$ with nonempty interior, there exists a finite set $\Lambda(\varepsilon, K)=\Lambda \subset E$, so that

$$
\sup _{x \in K}|p(x)| \geqq(1-\varepsilon)\|p\|_{\infty}, \quad \text { for all } p \in C_{E \backslash \Lambda}(G) .
$$

Detailed studies of properties (D) and (D1) and their relationships to Sidon sets appear in [3], [4], and [5].

THeOREM A (M. DÉCHAMPS-Gondim). If $\Gamma$ is torsion free, and $E \subset \Gamma$ is a Sidon set, then $E$ satisfies property (D).

Received by the editors May 23, 1972.

AMS (MOS) subject classifications (1969). Primary 4250, 4252.

Key words and phrases. Sidon set.

(c) American Mathematical Society 1973 
THEOREM B. Let $\Gamma$ be a discrete abelian group and $E \subset \Gamma$ a non-Sidon set. Then there exists a non-Sidon set $F \subset E$ so that $F$ satisfies property (D1).

Let $\Gamma$ be a countable group. Then, $\hat{\Gamma}=G$ is a compact metrizable group, and therefore there exists $D$, a countable dense subgroup of $G$. Consider $D$ as a discrete abelian group, and let $\phi: \Gamma \rightarrow \hat{D}$ be the natural injective map: $(\phi(\gamma), d)=(\gamma, d)$, for $\gamma \in \Gamma$ and $d \in D$. We say that $F \subset \hat{D}$ is a Sidon set if $E$ is a Sidon set in $(\hat{D})_{d}$, $\hat{D}$ with the discrete topology. Our proof of Theorem B is based on the following two lemmas, for whose proof we refer to Lemmas 2.2 and 2.3 in [1].

Lemma 3. Let $\Gamma, D$, and $\phi$ be as above. Then $E \subset \Gamma$ is a Sidon set if and only if $\phi(E)$ is Sidon.

LEMma 4. Let $E \subset \Gamma$ be a non-Sidon set. Then there exists a non-Sidon set $F \subset E$, so that $\overline{\phi(F)}$ (closure in $\hat{D})$ is a countable set with one limit point.

Proof of theorem B. We first assume that $\Gamma$ is a countable group, and we let $D$ and $\phi$ be as above. By Lemma 4, we choose a non-Sidon set $F \subset E$ so that $\phi(F)$ accumulates only at $x_{0} \in \hat{D}$, and without loss of generality we assume $x_{0}=0$. Suppose $\varepsilon>0$ is given, and $K \subset G$ is a compact neighborhood of 0 . By the density of $D$ in $G$, and by the compactness of $G$, we select $S=\left\{d_{j}\right\}_{j=1}^{N} \subset D$, so that $\left\{K+d_{j}\right\}_{j=1}^{N}$ is a covering for $G$. By a theorem of Wiener (e.g., cf. $[1,1.3])$, we find $V$, a neighborhood of 0 in $\hat{D}$, so that if $\mu \in M(V)$ then $\left|\hat{\mu}(d)-\hat{\mu}\left(d^{\prime}\right)\right|<(\varepsilon / 2)\|\hat{\mu}\|_{\infty}$, whenever $d-d^{\prime} \in S$. Let $\Lambda \subset F$ be a finite set so that $\phi(F \backslash \Lambda) \subset V$. Suppose $p$ is a trigonometric polynomial in $C_{F \backslash \Lambda}(G), p(g)=\sum_{j=1}^{M} a_{j}\left(\gamma_{j}, g\right)$, and $\|p\|_{\infty}=1$. We can consider $\hat{p}$ as a discrete measure on $\hat{D}: \hat{p}=\mu=\sum_{j=1}^{M} a_{j} \delta_{\phi\left(\gamma_{j}\right)}$, and $\|\hat{\mu}\|_{\infty}=1$. We find $g_{0} \in G$ so that $\left|\sum_{j=1}^{M} a_{j}\left(\gamma_{j}, g_{0}\right)\right|=1$, and let $g \in K$ be so that $g_{0}=g+d_{j_{0}}$, where $d_{j_{0}} \in S$. By the density of $D$ in $G$, we find $d^{\prime} \in K \cap D$, so that

$$
\left|1-\sum_{j=1}^{M} a_{j}\left(\gamma_{j}, d^{\prime}+d_{j_{0}}\right)\right|<\varepsilon / 2 .
$$

But, by the choice of $V$,

$$
\left|\sum_{j=1}^{M I} a_{j}\left(\phi\left(\gamma_{j}\right), d^{\prime}\right)-\sum_{j=1}^{M} a_{j}\left(\phi\left(\gamma_{j}\right), d^{\prime}+d_{j_{0}}\right)\right|<\varepsilon / 2,
$$

and therefore,

$$
1-\varepsilon \leqq\left|\sum_{j=1}^{M} a_{j}\left(\gamma_{j}, d^{\prime}\right)\right| \leqq \sup _{x \in K^{\prime}}|p(x)| .
$$

If $\Gamma$ is a discrete abelian group, and $E \subset \Gamma$ is a non-Sidon set, then there exists a countable non-Sidon set $E^{\prime} \subset E$. It easily follows that $S \subset g p\left(E^{\prime}\right)$ 
is a Sidon set in $\Gamma$ if and only if $S$ is a Sidon set in $g p\left(E^{\prime}\right)$. Therefore, there exists a non-Sidon set $F \subset E$, so that $F$ satisfies property (D1) with respect to $g p\left(E^{\prime}\right)^{\wedge}$. Let $\varepsilon>0$ be given, and $K$ be any compact neighborhood of 0 in $G$. The canonical map $\tau: G \rightarrow G / g p\left(E^{\prime}\right)^{\perp}$ is an open map, and therefore we can find a finite set $\Lambda \subset F$, so that

$$
\sup _{x \in \tau(K)}|g(x)| \geqq(1-\varepsilon)\|g\|_{\infty}, \quad \text { for all } g \in C_{F \backslash \Lambda}\left(g p\left(E^{\prime}\right)^{\wedge}\right) \text {. }
$$

But, if $p \in C_{F \backslash \Lambda}(G)$, then $p(x+y)=p(x)$, for all $x \in G, y \in g p\left(E^{\prime}\right)^{\perp}$, and

$$
\sup _{x \in K}|p(x)| \geqq(1-\varepsilon)\|p\|_{\infty} .
$$

REMARK. We say that a set $E \subset \Gamma$ has infinite pace, if given any finite set $F \subset \Gamma, F+E \cap E$ is a finite set. It is a consequence of a theorem of Wiener (cf. proof of Theorem B) that if $E \subset \Gamma$ satisfies property (D1) and $G$ is connected, then $E$ has infinite pace. We do not know whether the converse is true.

ADDED IN PROOF. Professor J.-P. Kahane indicated to us that the converse is false: There exists $E \subset Z$ so that $E$ has infinite pace, and $E$ violates property $(\mathrm{D})$.

\section{REFERENCES}

1. R. C. Blei, On trigonometric series associated with separable, translation invariant subspaces of $L^{\infty}(G)$, Trans. Amer. Math. Soc. (to appear).

2. M. Déchamps-Gondim, Compacts associés à un ensemble de Sidon, C. R. Acad. Sci. Paris Sér. A-B 271 (1970), A590-A592. MR 42 \#6526.

3. —, Ensembles de Sidon topologiques, Ann. Inst. Fourier (Grenoble), 22, (1972).

4. J. F. Méla, Sur certains ensembles exceptionnels en analyse de Fourier, Ann. Inst. Fourier (Grenoble) 18 (1968), 31-71.

5. K. A. Ross, Fatou-Zygmund sets (to appear).

6. W. Rudin, Fourier analysis on groups, Interscience Tracts in Pure and Appl. Math., no. 12, Interscience, New York, 1962: MR 27 \#2808. 06268

Department of Mathematics, University of Connecticut, Storrs, Connecticut

Current address: Istituto di Matematica, Università di Genova, Genova, Italy 